\title{
PRESENÇA DE DISLIPIDEMIA E USO DE ESTATINAS EM TRABALHADORES DA AGROINDÚSTRIA E PRODUTORES RURAIS: ESTUDO PRELIMINAR
}

\author{
Caroline Glier ${ }^{1}$ \\ Éboni Marília Reuter ${ }^{2}$ \\ Polliana Radtke dos Santos ${ }^{3}$ \\ Valeriano Antonio Corbellini ${ }^{4}$ \\ Hildegard Hedwig Pohl $l^{5}$ \\ Miriam Beatrís Reckziegel
}

\begin{abstract}
RESUMO
Alterações no perfil lipídico estão relacionadas ao estilo de vida e comumente são tratadas com estatinas. Desta forma, esta pesquisa objetiva caracterizar trabalhadores da agroindústria e produtores rurais quanto à presença de dislipidemia e uso de estatinas, associado a variáveis demográficas. Foi realizado estudo transversal e analítico com 127 sujeitos da região do Conselho Regional de Desenvolvimento do Vale do Rio Pardo, avaliados em 2012-2013. O uso de estatinas, bem como as variáveis demográficas (sexo, faixa etária, estado civil, classe econômico e escolaridade), foram obtidas por questionários. A definição da presença ou ausência de dislipidemia foi definida pelos marcadores bioquímicos obtidos através de coleta sanguínea após 12 horas de jejum. Os dados foram analisados com medidas de frequência e percentual, além do teste Exato de Fisher ( $\mathrm{p}<0,05$ ). Constatou-se que 61,4\% dos sujeitos eram dislipidêmicos, dos quais $89,7 \%$ não usavam estatinas. Houveram diferenças demográficas quanto à faixa etária entre aqueles que usam ou não estatinas, tanto na presença de dislipidemia $(\mathrm{p}=0,006)$ quanto em sua ausência $(\mathrm{p}=0,022)$. Verificou-se o desconhecimento e falta de controle dos níveis do perfil lipídico por alguns indivíduos.
\end{abstract}

Palavras-chave: Dislipidemias. Inibidores de Hidroximetilglutaril-CoA Redutases. Saúde do Trabalhador. Estilo de vida.

\begin{abstract}
Changes in lipid profiles are related to lifestyle and are commonly treated with statins. This study aims at characterizing agroindustry workers and rural producers regarding the presence of dyslipidemia and statins, associated with demographic variables. A cross-sectional and analytical study was performed with 127 subjects in the region of Rio Pardo Valley Development Regional Council, evaluated in 2012-2013. Statin use and demographic variables (gender, age, marital status, economic class and education) were obtained by questionnaires. The definition of the presence or absence of dyslipidemia was defined by biochemical markers, obtained from blood collection after 12 hours of overnight fasting. Data

\footnotetext{
1 Acadêmica do Curso de Fisioterapia da Universidade de Santa Cruz do Sul -UNISC. $<$ krol_glier@hotmail.com>

${ }^{2}$ Mestranda do Programa de Pós-Graduação em Promoção da Saúde da Universidade de Santa Cruz do Sul UNISC. <ebonir@outlook.com>

${ }^{3}$ Acadêmica do Curso de Fisioterapia da Universidade de Santa Cruz do Sul -UNISC. <polliana@mx2.unisc.br>

4 Professor do Departamento de Química e Física da Universidade de Santa Cruz do Sul -UNISC. <valer@unisc.br>

${ }_{5}$ Professor do Departamento de Educação Física e Saúde da Universidade de Santa Cruz do Sul -UNISC. $<$ hpohl@unisc.br>

${ }^{6}$ Professor do Departamento de Educação Física e Saúde da Universidade de Santa Cruz do Sul -UNISC. <miriam@unisc.br>.
} 
was analyzed with measures of frequency and percentage, in addition to Fisher's Exact test $(p<0.05)$. There were demographic differences in age between those who use statins or not, both in the presence of dyslipidemia $(p=0.006)$ and in its absence $(p=0.022)$. There was the lack of knowledge and control of the levels of lipid profile by some individuals.

Keywords: Dyslipidemias. Hydroxymethylglutaryl-CoA Reductase Inhibitors. Occupational Health. Life Style.

\section{INTRODUÇÃO}

A constante transformação do estilo de vida da população nos remete a importantes fatores de riscos cardiovasculares, uma vez que estas doenças são consideradas as principais causas de mortalidade no mundo (FALCÃO, 2011). Por apresentarem estreita associação com esta condição, as dislipidemias são o objeto de estudo de muitas pesquisas, sendo necessário compreender as alterações no metabolismo lipídico desencadeadas, bem como as possibilidades de tratamento.

Sabe-se que, nesta busca pelo controle da dislipidemia, as intervenções mais eficazes sem a utilização de medicamentos são as de menor adesão. Modificações no cotidiano - com inclusão de exercício físico, o abandono do tabagismo e a reeducação alimentar - são hábitos de baixa adesão quando comparados à ingestão de uma nova medicação. Dessa forma, a prescrição de hipolipemiantes com capacidade considerável para redução da hipercolesterolemia está se tornando uma prática frequente na clínica médica. Dentre os medicamentos que possuem este efeito encontram-se as estatinas, que diminuem a biossíntese de colesterol e possuem uma incidência considerável na redução de eventos cardiovasculares (XAVIER et al., 2013).

Ao representar um problema de saúde pública, os casos de dislipidemias devem ser tratados através de programas de prevenção e educação, sendo que a terapêutica desta doença deve iniciar com mudanças no estilo de vida, com hábitos alimentares saudáveis, manutenção ou aquisição de massa corporal adequada, exercícios físicos regulares, redução do tabagismo e bem estar emocional. Dentro deste contexto, é enfatizada a prática de exercícios físicos como fator essencial na prevenção e tratamento das dislipidemias (CAMBRI et al., 2006).

Sendo assim o presente trabalho tem como objetivo caracterizar trabalhadores da agroindústria e produtores rurais quanto à presença de dislipidemia e uso de estatinas, associando a variáveis demográficas. 


\section{FUNDAMENTAÇÃO TEÓRICA}

As dislipidemias caracterizam-se por alterações no metabolismo dos lipídeos, as quais se definem por uma elevação na concentração de lipoproteínas aterogênicas (LDL-c) e de triglicerídeos (TGs), e uma diminuição nos níveis plasmáticos da lipoproteína de alta densidade (HDL-c). Podendo ser classificadas como primárias (genética, alterações neuroendócrinas e distúrbios metabólicos) ou secundárias (diabetes mellitus, alcoolismo, obesidade, sedentarismo, dieta desequilibrada) (FALCÃO, 2011; FERREIRA, 2011; CARDOSO, 2011).

Segundo a proposição da V Diretriz Brasileira Sobre Dislipidemias e Prevenção da Aterosclerose (XAVIER et al., 2013), somente um marcador bioquímico alterado (LDL-c, TG, ou HDL-c) já permite a classificação do indivíduo como dislipidêmico. No entanto, conforme a associação ou não dessas modificações nas concentrações séricas, pode-se definir os tipos existentes de dislipidemias. Caso haja somente a elevação do LDL-c ( $\geq 160 \mathrm{mg} / \mathrm{dl})$, considera-se como hipercolesterolemia isolada. Se houver aumento isolado dos TGs $(\geq 150$ $\mathrm{mg} / \mathrm{dl}$ ), têm-se uma hipertrigliceridemia isolada. No entanto, se forem detectados valores elevados de LDL-c ( $\geq 160 \mathrm{mg} / \mathrm{dl}$ ) e TGs $(\geq 150 \mathrm{mg} / \mathrm{dl}$ ), classifica-se como hiperlipidemia mista. E se apresentar uma redução do HDL-c (homens < $40 \mathrm{mg} / \mathrm{dl}$ e mulheres $<50 \mathrm{mg} / \mathrm{dl}$ ) isolada ou em associação ao aumento de LDL-C ou de TG, define-se como HDL-c baixo.

Dessa forma, pode-se assimilar tais mudanças metabólicas como consequência de fatores genéticos, ambientais ou mesmo a associação destes (FREITAS, 2011). Conforme Falcão (2011), a genética, o sexo e a faixa etária são elementos relevantes no aparecimento de dislipidemia, porém há que se considerar as constantes transformações da sociedade, que acarretam um novo estilo de vida. Ou seja, uma alimentação com deficiências nutricionais, associada ao sedentarismo, são práticas que passaram a fazer parte do cotidiano da população e extremamente prejudiciais para uma vida saudável.

Além disso, Ferreira et al (2011) salientam que o excesso de peso, em especial a obesidade, produz diferentes modificações no metabolismo, como a dislipidemia, fatos que predispõem ao surgimento de patologias cardiovasculares. Dentre inúmeras doenças a aterosclerose apresenta um impacto importante, uma vez que as dislipidemias, principalmente as hiperlipidemias, ocasionam um aumento na formação das placas ateroscleróticas, produzindo uma cascata de reações negativas no organismo do indivíduo, que consequentemente leva à morte tecidual (MILLER, 1994, apud CARDOSO, 2011). 
Sendo assim, é indicado que o controle da dislipidemia pode ser realizado através de uma terapia nutricional e modificações no estilo de vida como, por exemplo, perda de peso, atividade física e cessação do tabagismo (XAVIER et al., 2013). Porém, à falta de adesão a estas mudanças, ou mesmo em virtude de outros fatores, as estatinas vêm sendo utilizadas no tratamento desta doença, por atuarem inibindo a ação competitiva da HMG CoA redutase, enzima limitante da síntese do colesterol, condicionando uma redução do LDL-c e lipoproteínas de densidade muito baixa (VLDL) (FREITAS, 2011).

As estatinas são drogas usadas no tratamento das dislipidemias, com estudos comprovando sua ação na redução da mortalidade por doença cardiovascular (DCV). Seus benefícios são independentes de sexo ou idade, sendo usados tanto na prevenção primária quanto na secundária de DCV (GAMA et al., 2005). A partir de dados apresentados por Bonfim et al (2013), pode se perceber que as estatinas são os principais medicamentos adotados por reduzirem o LDL-c de $15 \%$ a $55 \%$, os TG de $7 \%$ a $28 \%$, e elevarem o HDL-c de $2 \%$ a $10 \%$. No entanto, apesar de serem recursos que devem ser utilizados pelo paciente, pois em alguns casos somente a mudança no estilo de vida não é o suficiente, inúmeras barreiras aparecem frente à adesão ao tratamento. Entre estas barreiras podem ser destacados os efeitos colaterais, o valor da medicação e a falta de informação (SANTOS, 2013). Porém, a eficiência e a segurança, fazem com que esta seja, atualmente, um dos medicamentos mais comercializados em todo o mundo (BONFIM et al, 2013).

\section{MATERIAIS E MÉTODOS}

Trata-se de estudo transversal e analítico, realizado com 127 trabalhadores da agroindústria e produtores rurais provenientes da cidade de Santa Cruz do Sul e de municípios da Microrregião Sul do Conselho Regional de Desenvolvimento (COREDE) do Vale do Rio Pardo (Vale Verde, Passo do Sobrado, Candelária, Rio Pardo, Pantano Grande e General Câmara) - RS. Vale ressaltar que esta amostra faz parte da Pesquisa Triagem de fatores de risco relacionados ao excesso de peso em trabalhadores da agroindústria usando novas tecnologias analíticas e de informação em saúde, previamente aprovada pelo Comitê de Ética em Pesquisa com Seres Humanos da Universidade de Santa Cruz do Sul (UNISC) sob protocolo número 2509/10.

Os dados foram coletados durante o período de 2012 e 2013 através de um questionário, previamente validado por Pohl et al. (2010), enfocando as seguintes questões: 
sexo (masculino ou feminino), faixa etária (dividido pela mediana em $<53$ e $\geq 53$ anos) e estado civil (dicotomizado em casado e não casado). Neste questionário também foi avaliada a classificação econômica, utilizando a proposição da Associação Brasileira de Empresas de Pesquisa (ABEP, 2013), em que as classes econômicas (A1, A2, B1, B2, C1, C2, D e E) são estabelecidas a partir de uma pontuação obtida conforme as características domiciliares (presença e quantidade de alguns itens domiciliares de conforto e grau escolaridade do chefe de família). No entanto, neste estudo, a classificação dos indivíduos ficou restrita as classes B, $\mathrm{C}$ e $\mathrm{D}$, sendo as duas últimas unidas devido ao número de sujeitos em cada classe.

Para definição de escolaridade foi aplicado o questionário sobre fatores de risco cardiovascular, do projeto Corações do Brasil (SBC, 2004), sendo definidos três níveis: $1^{\circ}$ nível - analfabeto ou primeiro grau incompleto; $2^{\circ}$ nível - primeiro grau completo ou segundo incompleto; e $3^{\circ}$ nível - segundo grau completo, superior incompleto ou completo.

A utilização ou não de medicação, neste caso da estatina, foi obtida no dia da avaliação física, momento que os sujeitos trouxeram os medicamentos de uso frequente para registro do princípio ativo e posologia, sendo que na organização dos dados para este estudo foi definida a presença ou ausência do uso de estatinas. Para a definição da presença ou ausência de dislipidemia foram considerados os marcadores bioquímicos, obtidos através de coleta sanguínea por punção venosa antecubital, após 12 horas de jejum noturno. A classificação foi realizada a partir dos parâmetros adotados pela V Diretriz Brasileira Sobre Dislipidemias e Prevenção da Aterosclerose (XAVIER et al., 2013), os quais compreendem: elevação isolada do LDL-C ( $\geq 160 \mathrm{mg} / \mathrm{dL}$ ); elevação isolada dos TG ( $\geq 150 \mathrm{mg} / \mathrm{dL}$ ); valores aumentados de ambos LDL-C $(\geq 160 \mathrm{mg} / \mathrm{dL})$ e TG $(\geq 150 \mathrm{mg} / \mathrm{dL})$; redução do HDL-C (homens $<40 \mathrm{mg} / \mathrm{dL}$ e mulheres $<50 \mathrm{mg} / \mathrm{dL}$ ) isolada ou em associação com aumento de LDL$\mathrm{C}$ ou de TG, sendo considerado dislipidêmico aquele que possuía ao menos uma das alterações acima referidas.

No primeiro momento, os sujeitos foram divididos em dois grupos: dislipidêmicos e não dislipidêmicos. Em seguida, cada grupo foi dividido conforme a utilização ou não de estatinas. As diferenças do perfil lipídico foram avaliadas quanto aos dados demográficos (sexo, faixa etária, estado civil, classe econômica e escolaridade). Os dados foram tabulados e analisados através do programa Statistical Package for Social Sciences for Windows (SPSS versão 20.0), utilizando medidas de frequência e percentual, além do teste Exato de Fisher, considerando-se significativo $\mathrm{p}<0,05$. 


\section{RESULTADOS E DISCUSSÃO}

Os resultados apontam que dos 127 sujeitos avaliados, 78 (61,4\%) são dislipidêmicos, sendo que, destes, 89,7\% não faziam uso de estatinas. Este resultado aponta que a maioria não faz o controle desta patologia, possivelmente por desconhecimento da presença da doença. Por outro lado, 12,2\% dos sujeitos não dislipidêmicos fazem uso de estatina. Quando analisada a presença de dislipidemia e a utilização de estatina, não houve diferenças entre os sexos; ambos os grupos apresentaram altos índices de dislipidêmicos que não utilizam medicação de controle ( 91,3 e 89,1\%, respectivamente, em homens e mulheres) (Tabela 1). Souza et al (2003) encontraram valores significativamente maiores no sexo masculino quando em comparação ao feminino, dado que contrapõe os resultados apresentados neste estudo, uma vez que, quando comparados, as mulheres apresentaram maior índice de dislipidemia em relação aos homens, possivelmente pelo fato desta amostra ser constituída principalmente pelo sexo feminino.

Com relação às faixas etárias, todos os sujeitos com idades inferiores a 53 anos não faziam uso de estatinas, mesmo com a presença de dislipidemia. Na faixa etária $\geq 53$ anos, a maioria dos dislipidêmicos $(80,5 \%)$ não utiliza estatina. A dislipidemia e o uso de estatinas apresentaram diferenças significativas quando consideradas a variável faixa etária, tanto nos sujeitos com dislipidemia ( $\mathrm{p}=0,006)$ quanto nos com ausência desta alteração metabólica $(\mathrm{p}=0,022)$.

Tabela 1 - Comparativo sobre o uso ou não de estatinas dividida entre dislipidêmicos ou não e relacionados ao sexo, idade e estado civil.

\begin{tabular}{|c|c|c|c|c|c|c|}
\hline \multirow{2}{*}{ Variáveis } & \multicolumn{3}{|c|}{$\begin{array}{c}\text { Presença Dislipidemia } \\
\mathrm{n}=78(61,4 \%)\end{array}$} & \multicolumn{3}{|c|}{$\begin{array}{c}\text { Ausência Dislipidemia } \\
n=49(38,6 \%)\end{array}$} \\
\hline & $\begin{array}{c}\text { Uso estatina } \\
\mathrm{n}(\%)\end{array}$ & $\begin{array}{c}\text { Não-uso } \\
\mathrm{n}(\%)\end{array}$ & $\mathrm{P}$ & $\begin{array}{c}\text { Uso estatina } \\
\mathrm{n}(\%)\end{array}$ & $\begin{array}{c}\text { Não-uso } \\
\text { n }(\%)\end{array}$ & $\mathrm{P}$ \\
\hline Total & $8(10,3)$ & $70(89,7)$ & & $6(12,2)$ & $43(87,8)$ & \\
\hline \multicolumn{7}{|l|}{ Sexo } \\
\hline $\begin{array}{l}\text { Masculino } \\
\text { Feminino }\end{array}$ & $\begin{array}{c}2(8,7) \\
6(10,9)\end{array}$ & $\begin{array}{l}21(91,3) \\
49(89,1)\end{array}$ & 1,000 & $\begin{array}{c}4(16,0) \\
2(8,3)\end{array}$ & $\begin{array}{l}21(84,0) \\
22(91,7)\end{array}$ & 0,667 \\
\hline \multicolumn{7}{|l|}{ Faixa etária } \\
\hline $\begin{array}{l}<53 \text { anos } \\
\geq 53 \text { anos }\end{array}$ & $8(19,5)$ & $\begin{array}{l}37(100,0) \\
33(80,5)\end{array}$ & $0,006 *$ & $6(24,0)$ & $\begin{array}{c}24(100,0) \\
19(76,0)\end{array}$ & $0,022 *$ \\
\hline \multicolumn{7}{|l|}{ Estado civil } \\
\hline $\begin{array}{l}\text { Casado } \\
\text { Não casado }\end{array}$ & $\begin{array}{c}6(9,4) \\
2(14,3)\end{array}$ & $\begin{array}{l}58(90,6) \\
12(85,7)\end{array}$ & 0,629 & $\begin{array}{l}4(11,1) \\
2(15,4)\end{array}$ & $\begin{array}{l}32(88,9) \\
11(84,6)\end{array}$ & 0,650 \\
\hline
\end{tabular}

Segundo a Sociedade Brasileira de Endocrinologia e Metabologia (2013), a dislipidemia é mais frequente nos homens com mais de 45 anos e em mulheres com mais de 
55 anos. Dados epidemiológicos demonstram que a mortalidade por doenças cardiovasculares aumenta conforme a idade dos indivíduos (BRANDÃO, 2004). No estudo de Souza et al (2003) foi observado um aumento na ocorrência de dislipidemia com a idade, sendo a maior prevalência na faixa de 50 a 59 anos, havendo decréscimo nas faixas etárias superiores.

Observou-se, conforme Tabela 1, que o estado civil não apresentou diferenças na distribuição de uso ou não de estatinas nos dois grupos (presença e ausência de dislipidemia) para ambas as categorias (casados e não casados), uma vez que nesta variável a grande maioria não faz uso de estatinas, independente da presença ou não da dislipidemia (Tabela 1). A partir da análise da Tabela 2, verificou-se que o uso ou não de estatinas, dividida entre dislipidêmicos ou não, relacionado à classe socioeconômica, não apresentou relação, uma vez que em todas as classes a maioria dos dislipidêmicos não utilizava estatinas (91,4 e 88,4\%, respectivamente, para classe $\mathrm{B}$ e classe $\mathrm{C}$ e D), assim como os trabalhadores com ausência de dislipidemia (classe B: 87,0; classe C-D: 88,5\%).

Tabela 2 - Comparativo sobre o uso ou não de estatinas dividida entre dislipidêmicos ou não estratificados por classe econômica e escolaridade.

\begin{tabular}{lcccccc}
\hline & \multicolumn{3}{c}{$\begin{array}{c}\text { Presença Dislipidemia } \\
\mathrm{n}=78(61,4 \%)\end{array}$} & \multicolumn{2}{c}{$\begin{array}{c}\text { Ausência Dislipidemia } \\
\mathrm{n}=49(38,6 \%)\end{array}$} \\
\cline { 2 - 7 } Variáveis & $\begin{array}{c}\text { Uso estatina } \\
\mathrm{n}(\%)\end{array}$ & $\begin{array}{c}\text { Não-uso } \\
\mathrm{n}(\%)\end{array}$ & $\mathrm{p}$ & $\begin{array}{c}\text { Uso estatina } \\
\mathrm{n}(\%)\end{array}$ & $\begin{array}{c}\text { Não-uso } \\
\mathrm{n}(\%)\end{array}$ & $\mathrm{p}$ \\
\hline Classe econômica & & & & & & \\
B & $3(8,6)$ & $32(91,4)$ & 0,724 & $3(13,0)$ & $20(87,0)$ & 1,000 \\
C-D & $5(11,6)$ & $38(88,4)$ & & $3(11,5)$ & $23(88,5)$ & \\
Escolaridade & & & & $5(14,7)$ & $29(85,3)$ & \\
Primeiro Nível & $5(9,4)$ & $48(90,6)$ & & $0(0,0)$ & $4(100,0)$ & 0,677 \\
Segundo Nível & $0(0,0)$ & $8(100,0)$ & 0,664 & $1(10,0)$ & $9(90,0)$ & \\
Terceiro Nível & $1(8,3)$ & $11(91,7)$ & & & &
\end{tabular}

Quanto à escolaridade, notou-se que grande parte da amostra pertencia ao primeiro nível, porém, independente desta classificação, a maioria dos dislipidêmicos não fazem uso de estatina (primeiro nível: 90,6\%; segundo nível: 100,0\%; terceiro nível: 91,7\%). O estudo de Moraes, Checchio e Freitas (2013) apresentou a prevalência de dislipidemia dividida por sexo e comparada a partir de dados sociodemográficos, sendo observado que, independente do sexo, a ocorrência de dislipidemia aumentou com a idade e reduziu com o aumento do número de anos investidos em educação formal.

As estatinas são consideradas um método terapêutico de grande aceitação no tratamento das dislipidemias. Porém, sabe-se da importância de um aprimoramento no uso destes fármacos quanto aos efeitos colaterais existentes, como revelam estudos que enfocam 
esta questão, mesmo diante dos efeitos destes medicamentos sobre o perfil lipídico (MAGALHÃES et al., 2004).

\section{CONCLUSÃO}

Os resultados apontam a presença de dislipidemias na maioria dos trabalhadores da agroindústria e produtores rurais, os quais, em grande número, não faziam uso de estatinas para o controle desta patologia. Este fato pode estar relacionado com o desconhecimento da presença da doença e a falta de cuidado com a alimentação e a prática de exercícios físicos, além da dificuldade de acesso ao atendimento nas unidades de saúde.

No que se refere à presença de dislipidemia e utilização de estatinas, houve relação significativa com a faixa etária, indicando que os sujeitos com menos de 53 anos não faziam uso de estatinas, mesmo com a presença de dislipidemias. Nas demais variáveis analisadas, como sexo, estado civil, classe econômica e escolaridade, essa relação não ficou evidente. Sugere-se aprofundamento de estudos dessa natureza a fim de otimizar o controle das dislipidemias através do manejo correto dos medicamentos e, principalmente, de intervenções não medicamentosas, bem como tornar visível este perfil, o que propiciará que políticas públicas, direcionadas a esta população, sejam implementadas.

\section{AGRADECIMENTOS}

À Universidade de Santa Cruz do Sul - UNISC, à Secretaria de Ciência, Inovação e Desenvolvimento Tecnológico do Estado do Rio Grande do Sul (SCIDT/RS), ao Polo de Modernização Tecnológica do Vale do Rio Pardo, à Fundação de Amparo à Pesquisa do Estado do Rio Grande do Sul (FAPERGS/RS), ao Conselho Nacional de Desenvolvimento Científico e Tecnológico (CNPq) e ao Programa de Pós-Graduação em Promoção da Saúde (PPGPS/UNISC). 


\section{REFERÊNCIAS}

ABEP. Associação Brasileiras de Empresas de Pesquisa. Critérios de Classificação Econômica - Brasil, 2013.

BONFIM, M. R.; OLIVEIRA, A. S. B.; AMARAL, S. L.; MONTEIRO, H. L. Caracterização do tratamento medicamentoso com estatinas em unidade básica de saúde. Medicina, Ribeirão Preto, v. 46, n. 1, p. 47-55, 2013.

BRANDÃO, A. A. Dislipidemias em idosos. Revista da SOCERJ, Rio de Janeiro, v. 17, s. B, p. 11-13, 2004.

CAMBRI, L. T.; SOUZA, M.; MANNRICH, G.; CRUZ, R. O.; GEVAERD, M. S. Perfil lipídico, dislipidemias e exercícios físicos. Revista Brasileira de Cineantropometria \& Desempenho Humano, Florianópolis, v.8, n. 3, p. 100-106, 2006.

CARDOSO, A. P. Z.; NOGUEIRA, M. S.; HAYASHIDA, M.; SOUZA, L.; CESARINO, E. J. Aspectos clínicos e socioeconômicos das dislipidemias em portadores de doenças cardiovasculares. Physis Revista de Saúde Coletiva, Rio de Janeiro, v. 21, n. 2, p. 417-436, 2011.

FALCÃO, R. A. Avaliação do uso de estatinas em pacientes dislipidêmicos. Trabalho de Conclusão de Curso (Curso de Graduação em Farmácia) - Universidade Estadual da Paraíba, Centro de Ciências Biológicas e da Saúde. Campina Grande - PB, 2011.

FERREIRA, N. L.; RODRIGUES, M. T. G.; ABREU, M. N. S.; LOPES, A. C. S. Fatores nutricionais associados às dislipidemias em usuários de Serviço de Atenção Primária à Saúde. Acta Médica Portuguesa, v. 24, n. S2, p. 457-466, 2011.

FREITAS, L. F. F. Avaliação do consumo de estatinas em Portugal entre 2000 e 2009. Projeto de Pós-Graduação (Faculdade de Ciências da Saúde) - Universidade Fernando Pessoa, Porto, 2011.

GAMA, M. P. R.; PELlEGRINELlO, S.; ALONSO, S. S. Q.; COELHO, J. F.; MARTINS, C. F. L.; BIAGINI, G. L. K. Rabdomiólise Devido ao Uso de Estatina em Altas Doses: Relato de Caso. Arquivos Brasileiros de Endocrinologia e Metabologia, Curitiba, v. 49, n. 4, 2005.

MAGAlHÃES, M. E. C.; BRANDÃO, A. A.; FREITAS, E. V.; POZZAN, R.; BRANDÃO, A. P. Novas Perspectivas no Tratamento das Dislipidemias. Revista da SOCERJ, Rio de Janeiro, v. 17, n. 2, p. 105-111. 2004.

MORAES, S. A.; CHECCHIO, M. V.; FREITAS, I. C. M. Dislipidemia e fatores associados em adultos residentes em Ribeirão Preto, SP: resultados do Projeto EPIDCV. Arquivos Brasileiros de Endocrinologia e Metabologia, v. 57, n. 9, p. 691-701, 2013.

POHL, H. H.; RECKZIEGEL, M. B.; VITIELlO, I. P., GALliANO, L. M. Saúde do trabalhador e estilo de vida: uma visão multisetorial da aptidão física. FIEP Bulletin, v. 80, Special Edition - Article I, 2010. 
SANTOS, M. C. B. Adesão ao tratamento com estatinas. Dissertação de Mestrado (Faculdade de Ciências da Saúde) - Universidade de Brasília, Brasília, Distrito Federal, 2013.

SBC. SOCIEDADE BRASILEIRA DE CARDIOLOGIA. Questionário sobre fatores de risco cardiovascular. Projeto Corações do Brasil, 2004.

SBEM. Sociedade Brasileira de Endocrinologia e Metabologia. Dislipidemia e Aterosclerose. [2013?]. Disponível em: <http://www.endocrino.org.br/dislipidemia-e-aterosclerose/> Acesso em: 05 mar. 2014.

SOUZA, L. J.; SOUTO FILHO, J. T. D.; SOUZA, T. F.; REIS, A. F. F.; GICOVATE NETO, C.; BASTOS, D. A.; CÔRTES, V. A.; CHALITA, F. E. B.; TEIXEIRA, C. L. Prevalência de dislipidemia e fatores de risco em Campo do Goytacazes - RJ. Arquivos Brasileiros de Cardiologia, v. 81, n. 3, p.249-56, 2003.

XAVIER, H. T.; IZAR, M. C.; FARIA NETO, J. R.; ASSAD, M. H.; ROCHA, V. Z.; SPOSITO, A. C. et al. Sociedade Brasileira de Cardiologia. V Diretriz Brasileira Sobre Dislipidemias e Prevenção da Aterosclerose. Arquivos Brasileiros de Cardiologia, v. 101, n. 4, supl. 1, p.1-22, 2013. 